\title{
Bildgebung des hepatozellulären Karzinoms
}

\section{Imaging of hepatocellular carcinoma}

\author{
T. Lincke, D. Boll, C. Zech
}

\begin{tabular}{l|l}
\hline Übersicht & \\
\hline Einleitung & 295 \\
\hline Risikofaktoren und Epidemiologie & 295 \\
\hline $\begin{array}{l}\text { Prävention und Früherkennung } \\
\text { Pathophysiologie }\end{array}$ & 296 \\
\hline $\begin{array}{l}\text { Bildgebende Diagnostik } \\
\text { Wichtige Differenzialdiagnosen } \\
\text { in der zirrhotischen Leber } \\
\text { Klassifikation }\end{array}$ & 296 \\
\hline
\end{tabular}

\section{Einleitung}

Das hepatozelluläre Karzinom (HCC) ist der häufigste primär maligne Tumor der Leber. Der Erkrankungsgipfel liegt zwischen der 5. und 7. Lebensdekade. Das HCC ist die weltweit zweithäufigste zum Tode führende Krebserkrankung bei Männern und die sechsthäufigste zum Tode führende Krebserkrankung bei Frauen. Im Jahr 2012 wurden in der EU fast genauso viele Neuerkrankungen wie Todesfälle registriert (52000 vs. 48000) [1].

In ca. 90\% der Fälle entwickelt sich das HCC in der westlichen Welt stufenweise aus einer zirrhotischen Leber über die Bildung von Regeneratknoten, dysplastischen Knoten und dysplastischen Knoten mit fokaler Läsion. Es kann jedoch auch de novo entstehen.

\section{Zusammenfassung}

Das hepatozelluläre Karzinom (HCC) ist eine der häufigsten Ursachen tumorbedingter Todesfälle weltweit. Trotz der Verbesserungen in der Diagnostik und Therapie hat sich die Anzahl der Neuerkrankungen und der Todesfälle nicht verändert. Hauptrisikofaktoren des HCC sind Leberzirrhose (90\%), nicht alkoholische Fettleber und chronische Hepatitis-B-Infektion. Um HCC-verdächtige Veränderungen frühzeitig zu entdecken, sollten Risikopatienten alle 6 Monate sonografiert werden. Die Diagnose kann mit kontrastmittelunterstützten bildgebenden Verfahren wie CEUS („contrast-enhanced-ultrasound“), CE-MRI („contrast-enhanced-MRI“) und CE-CT („contrast-enhanced-CT“) gestellt werden. In diesem Artikel werden ihre charakteristischen Befunde beim HCC vorgestellt.
Wie die Hepatitis B ist auch das HCC geografisch
unterschiedlich verteilt. Am häufigsten ist es in Sü ostasien und Afrika. In den letzten Jahrzehnten hat jedoch die Inzidenz des HCC auch in den westlichen Industrieländern erheblich zugenommen, was auf das versetzte Auftreten chronischer HCV-Infektionen und die Zunahme der nicht alkoholischen Fettleberhepatitis („nonalcoholic fatty liver disease“, NAFLD) als Folge des metabolischen Syndroms und des Diabetes mellitus, zurückgeführt wird [3]. Ein Alkoholkonsum von mehr als $60-80 \mathrm{~g} / \mathrm{d}$ bei Männern bzw. mehr als $20 \mathrm{~g} / \mathrm{d}$ bei Frauen geht mit einem Zehnjahresrisiko für ein HCC 
von 6-41\% einher. Stoffwechselerkrankungen wie z.B. die hereditäre Hämochromatose, Aflatoxine und Rauchen gelten ebenfalls als Risikofaktoren für die HCCEntstehung [4, 5].

Die Leberzirrhose ist der wichtigste Risikofaktor für die HCC-Entstehung.

\section{Prävention und Früherkennung}

\section{Präventionsprogramme}

Aus den genannten Risikofaktoren lässt sich ableiten, dass die folgenden Präventionsprogramme sinnvoll sind:

- flächendeckende Hepatitis-B-Impfungen (weniger HBV-Infektionen haben insbesondere in Endemiegebieten zu einer Abnahme der Erkrankung bei Jugendlichen und jungen Erwachsenen geführt [6])

- Alkoholkarenz bei jeder chronischen Lebererkrankung

- antivirale Therapie bei Patienten mit chronischen Viruserkrankungen entsprechend den S3-Leitlinien der Deutschen Gesellschaft für Gastroenterologie, Verdauungs- und Stoffwechselkrankheiten (DGVS)

- Gewichtsreduzierung bei NAFLD bzw. NASH („,nonalcoholic steatohepatitis“, nicht alkoholische Steatohepatitis)

- Metformin-Therapie bei Patienten mit nicht insulinabhängigem Typ-2-Diabetes [7]

\section{Früherkennung}

Wenn die Diagnose im Rahmen eines Früherkennungsprogramms gestellt wird, kann eine 5-JahreÜberlebensrate von 50\% erreicht werden. Dagegen liegt sie bei nur $0-10 \%$, wenn bereits erste Symptome aufgetreten sind [8].

Sonografie. Die aktuelle S3-Leitlinie des HCC und die aktuellen EASL-Guidelines (EASL=European Association for the Study of the Liver) empfehlen halbjährliche Sonografien (konventioneller Ultraschall, nicht kontrastverstärkt, durchgeführt durch entsprechend qualifiziertes Personal) beim Hochrisikokollektiv. CT und MRT werden nicht empfohlen, weil bisher keine klinischen Studien bezüglich der Früherkennung eines HCC vorliegen. Ausnahme ist eine ergänzende Verwendung, wenn die Lebersonografie z. B. infolge einer ausgeprägten Darmgasüberlagerung nur eingeschränkt beurteilbar ist.
Tumormarker. Die Bestimmung von Tumormarkern wird für die Früherkennung derzeit nicht empfohlen. Das gilt sowohl für das AFP als auch für neuere Marker wie das AFP-L3 (lektinreaktives $\alpha$-Fetoprotein) oder das DCP („des-gamma-carboxy prothrombin“). Unabhängig davon werden einige dieser Marker aber für die Verlaufsbeurteilung verwendet.

\section{Pathophysiologie}

\section{Veränderungen während der Hepatokarzinogenese}

Die Leberläppchen sind die kleinste Struktureinheit der Leber. Zwischen ihnen liegen die Periportalfelder, die die Glisson-Trias beinhalten: Gallengang, Arterie und Vene, also Ductus biliferi interlobulares, Aa. interlobulares (Äste der A. hepatica propria) und Vv. interlobulares (Äste der Pfortader). Während der Hepatokarzinogenese kommt es mit zunehmender Tumorgröße und zunehmender Entdifferenzierung zu

- einer Zunahme der arteriellen Gefäße außerhalb dieser Glisson-Trias,

- einer Verringerung der Zahl der Periportalfelder sowie

- einer Zunahme der venösen Drainage über Lebersinusoide und Portalvenen (gegenüber Lebervenen in benigne Vorstufen) [9].

Diese Veränderungen erklären schließlich die u.g. Charakteristika des HCC.

\section{Einteilung}

Makroskopisch können 3 Wachstumsformen des HCC unterschieden werden: das solitäre bzw. fokal-noduläre HCC, das multinoduläre HCC und das diffus-infiltrative HCC. Je nach Tumorgröße und Typ sind die Läsionen scharf oder unscharf gegen das Leberparenchym abgrenzbar.

Die „international consensus group for hepatocellular carcinoma“ teilt das HCC darüber hinaus in eine frühe und eine progrediente Form ein [24]. Diese weisen die folgenden Charakteristika auf:

- frühes HCC: eher klein (<2 cm), annährend nodulär konfiguriert, gut differenziert

- progredientes HCC: eher groß (>2 cm), eindeutig nodulär konfiguriert, mäßig differenziert 


\section{Bildgebende Diagnostik}

\section{Diagnostische Kriterien}

Die Diagnostik des HCC beruht, unabhängig von der zugrunde liegenden Ätiologie, auf einem charakteristischen Perfusionsverhalten. Dies kann sowohl mit kontrastverstärktem Ultraschall (CEUS) als auch mit kontrastverstärkter CT (CE-CT) oder MRT (CE-MRT) nachgewiesen werden.

\section{Hauptkriterien}

Als bildgebend bewiesen gilt ein HCC bei Patienten mit Leberzirrhose entsprechend den EASL-Guidelines bzw. im Hochrisikokollektiv entsprechend der S3-Leitlinie, wenn eine Läsion in der

- arteriell-dominanten Phase eine Hypervaskularisierung („wash in“) und

- in der folgenden portalvenösen bis parenchymatösen Phase ein Auswaschphänomen („wash out“), z. T. bis hin zur Kontrastumkehr,

aufweist. Diese Hauptkriterien sind jedoch nicht bei jedem HCC nachweisbar.

Nur $80-85 \%$ der HCCs zeigen ein Wash-in und nur $50-70 \%$ der HCCs zeigen ein Wash-in und Washout.

\section{Weitere Kriterien}

Neben diesen Hauptkriterien gibt es weitere wichtige HCC-Charakteristika (Abb.1-5), die es zu beurteilen gilt.

Kapsel und Septen. Dabei handelt es sich um eine Tumorkapsel bzw. Pseudokapsel und um intratumorale fibröse Septen. Sie stellen sich meist länger kontrastreich dar als das Tumorgewebe (Abb.1) und finden sich häufig beim fortgeschrittenen HCC, gelegentlich aber auch in Regeneratknoten, dysplastischen Knoten und bei intrahepatischen cholangiozellulären Karzinomen (ICC).

Corona-Enhancement. Dieses peritumorale Enhancement (Abb.2) ist Folge des Kontrastmittelabstroms in die Lebersinusoide und setzt leicht verzögert gegenüber dem arteriellen Enhancement der HCC-Läsion ein. Ein Corona-Enhancement ist typisch für ein fortgeschrittenes HCC und erleichtert die Differenzierung zur AEP (,arterial enhancing pseudolesion“).
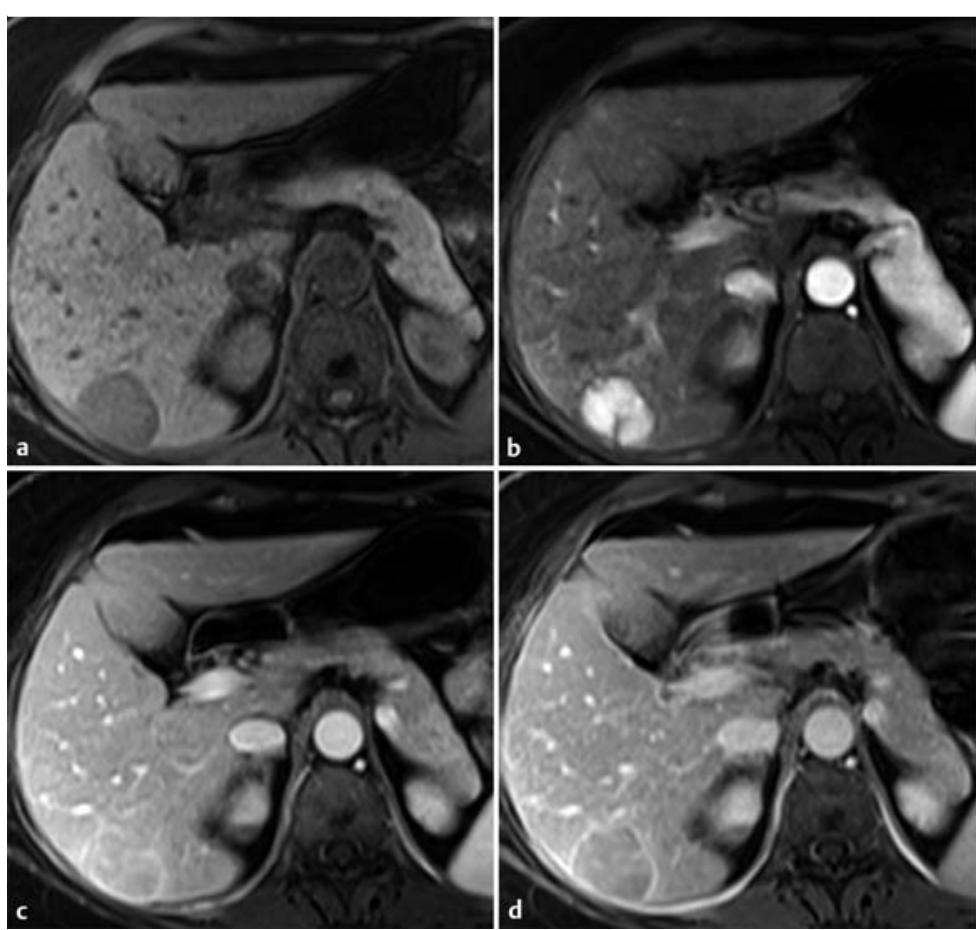

Abb. 1 Kapsel-Enhancement, T1w Fettsättigung. Der Nachweis eines Kapsel-Enhancements bzw. das Vorliegen einer Pseudokapsel ist ein wichtiges diagnostisches Kriterium des HCC. a Native Bildgebung zu Beginn. b - d Typischerweise setzt die Kapselkontrastierung verzögert gegenüber der Kontrastierung des Resttumorgewebes ein (b, früharteriell) und hält etwas länger an (c, portalvenös und d, spätvenös).

Mosaikartige Binnenstruktur. Ein Mosaik-Pattern (Abb.3) findet sich insbesondere bei größeren HCCLäsionen. Sie erleichtert die Differenzierung zum ICC.

Intraläsionales Fett. Fett innerhalb der Läsion (Abb.4) lässt eher auf ein frühes HCC schließen, findet sich gelegentlich jedoch auch im dysplastischen Knoten und fortgeschrittenem HCC.

Dysplastischer Knoten mit fokaler Läsion. Wenn sich ein kleines HCC innerhalb eines dysplastischen Knotens entwickelt, entsteht das Bild eines „nodule in a nodule“ (Abb.5): In der MR-Diagnostik zeigt sich innerhalb einer T2w hypointensen Läsion ein hyperintenser Fokus.

\section{Beurteilung weiterer Veränderungen}

\section{- Intrahepatisches Staging}

Ein HCC breitet sich vor allem intrahepatisch aus. Das primär organüberschreitende Wachstum mit Infiltration von Nachbarorganen ist dagegen selten. Häufig findet hingegen bei größeren Tumoren eine Gefäßinfiltration statt. Das intrahepatische Staging sollte deshalb die genaue Beschreibung der Größe und Lokalisation 

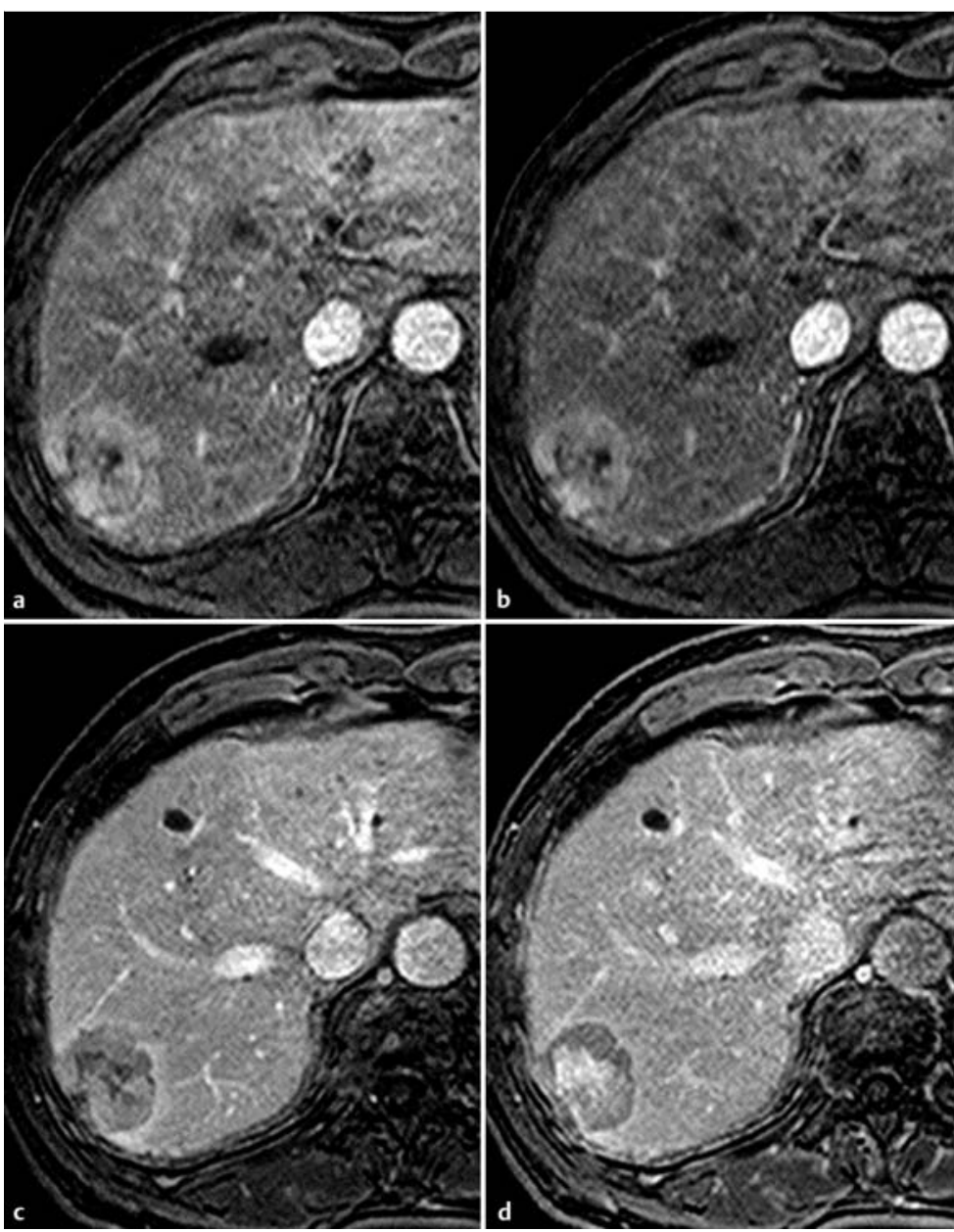

Abb. 2 Corona-Enhancement, T1w Fettsättigung. Das Corona-Enhancement ist ein wichtiges zusätzliches diagnostisches Kriterium in der Beurteilung des HCC. Es setzt etwas verzögert gegenüber dem arteriellen Enhancement der HCC-Läsion ein und ist Folge eines Kontrastmittelabstroms in die angrenzende Lebersinusoide. a Früharteriell. b Spätarteriell. c Portalvenös. d Spätvenös. sämtlicher Läsionen, das Vorhandensein und die Charakterisierung von Thrombosen der Portalvenenäste sowie die Infiltration der Lebervenen beinhalten. Außerdem sollte man beurteilen, ob arterioportale Shunts vorliegen. Sie führen zu einer frühen Kontrastierung der Portalvenen und spielen insbesondere bei einer geplanten interventionellen Embolisationsbehandlung (TACE oder SIRT) eine Rolle.

\section{- Pfortaderthrombosen}

Mitunter ist es schwierig, einen blanden Pfortaderthrombus von einem malignen Thrombus zu unterscheiden, der durch direkte Tumorinvasion entstanden ist. Diese Differenzierung ist jedoch für die weitere Therapieplanung entscheidend. Allgemeingültige Kriterien für Thrombosen wie der direkte Nachweis intraluminalen Materials, die Distension der Pfortader auf mehr als $13-15$ mm, das Fehlen der Atemvariabilität und letztlich das Fehlen eines Flusssignals innerhalb der Pfortader gelten sowohl für benigne als auch für maligne Thrombosen. Ein maligner Thrombus zeichnet sich insbesondere durch ein dem HCC äquivalentes Enhancement aus. Blande Thromben sind avaskularisiert und zeigen dementsprechend kein Enhancement und kein Wash-out. Zusätzliche Informationen liefert die Diffusionsbildgebung: Bei malignen Thromben sind die ADC-Werte infolge der erhöhten Zelldichte erniedrigt, bei benignen Thromben erhöht [25]. Die Ausbildung von Kollateralen mit kavernöser Transformation weist eher auf einen benignen Thrombus hin [26]. Gelegentlich wird zur ganz sicheren Charakterisierung eines Pfortaderthrombus auch eine bildgesteuerte Biopsie durchgeführt.
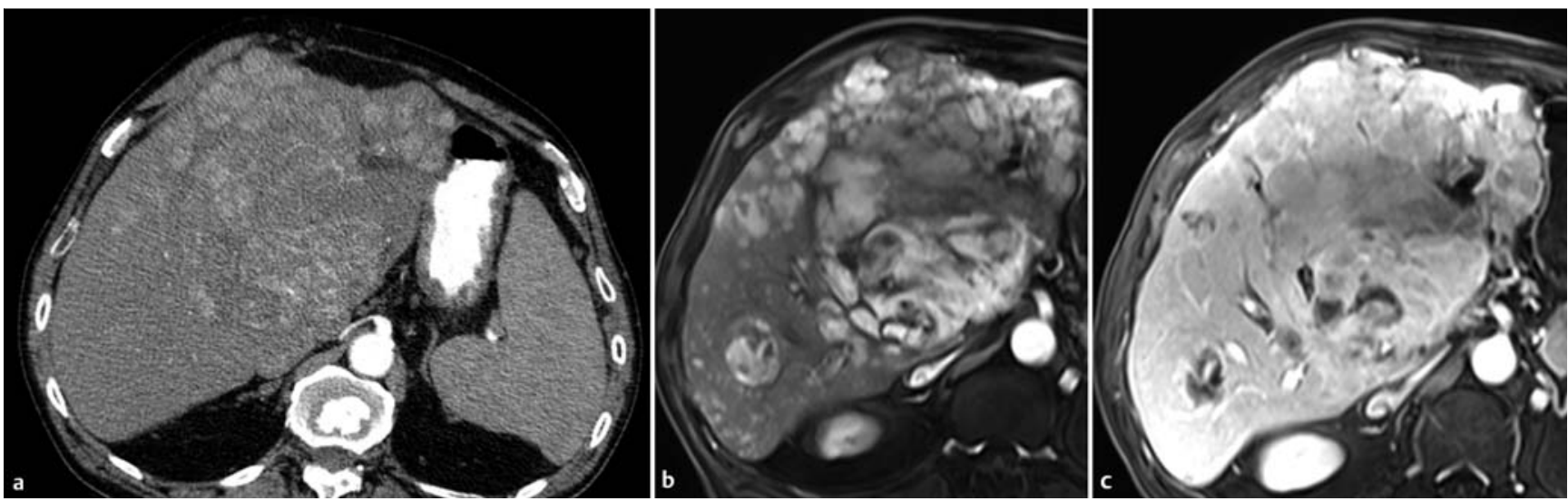

Abb. 3 Typisches Mosaik-Pattern einer großen, multilokulären HCC-Manifestation. a CT. b, c MRT (b, früharteriell und c, portalvenös, T1w Fettsättigung). 


\section{- Extrahepatisches Staging}

Das extrahepatische Staging ist insbesondere wichtig, wenn bereits ein ausgedehnter Tumorbefall und eine makroskopische Gefäßinvasion in der Leber vorliegen insbesondere im Hinblick auf die Auswahl einer geeigneten Therapie. Zu erwarten ist eine lymphogene Metastasierung in lokoregionäre Lymphknoten im Leberhilus. Vergrößerte Lymphknoten im Leberhilus werden aber auch regelmäßig bei Patienten mit Leberzirrhose ohne HCC beobachtet, sodass die Differenzierung zwischen reaktiven und neoplastischen Lymphknoten hier noch schwieriger ist als in anderen Bereichen. Gemeinhin wird in dieser Situation ein Lymphknoten erst als neoplastisch angesehen, wenn er im Verlauf eindeutig größer wird und/oder sein Durchmesser über $15 \mathrm{~mm}$ beträgt [10]. Die hämatogene Aussaat betrifft meist die Lunge, seltener auch die Nebennieren, das Peritoneum/Omentum und das Skelett.

\section{Methoden}

\section{- Sonografie}

HCC-Läsionen präsentieren sich im konventionellen Ultraschall je nach ihrer Größe häufig mit einem gemischten Echomuster, wobei kleinere HCC-Läsionen $(<3 \mathrm{~cm})$ meist hypoechogen zur Darstellung kommen. Die Hypervaskularisierung kann mittels Doppler- und Powerdopplersonografie gezeigt werden. Die arterielle Hypervaskularisierung und der anschließende rasche Wash-out können mit der kontrastverstärkten Sonografie dargestellt werden, HCCs lassen sich zuverlässig charakterisieren (Abb.6). Als weiterer Vorteil ist die Sonografie schnell verfügbar und kostengünstig. Einschränkungen sind die Untersucherabhängigkeit, die Notwendigkeit moderner Ultraschallgeräte, patientenbezogene Charakteristika wie Adipositas, Tumorcharakteristika wie die Tumorlage und letztlich auch das Vorhandensein einer Leberzirrhose, weil die Schallwellen dann weniger tief in die Leber vordringen können. Ebenso erfordert das Vorhandensein mehrerer Läsionen eine wiederholte Kontrastmittelgabe, was im Untersuchungsverlauf zu einer eingeschränkten Beurteilbarkeit der Läsionen führen kann.

\section{- Computertomografie}

HCC-Läsionen können mit der CT mit hoher Sicherheit nachgewiesen werden. Vorteile der CT sind die weite Verbreitung und schnelle Verfügbarkeit, die standardisierte Durchführung und die Robustheit gegenüber Artefakten. Etabliert ist ein triphasisches Untersuchungsprotokoll der Leber mit

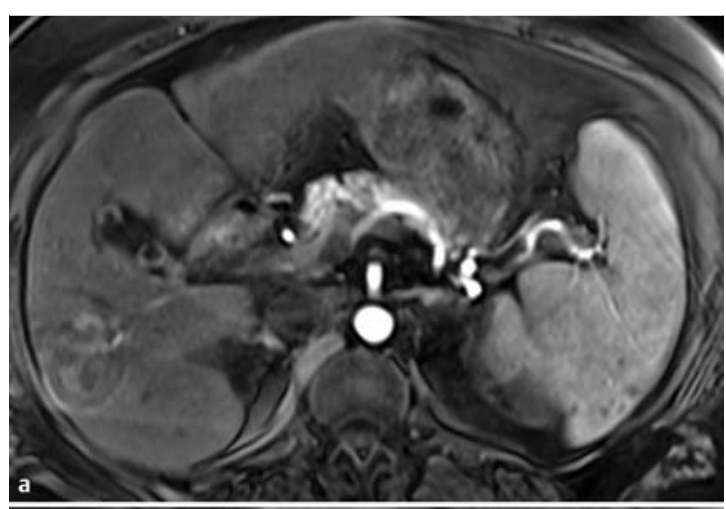

Abb. 4 Intraläsionales Fett. a In der T1w Fettsättigung kommt in der arteriellen Phase eine glatt berandete, zentral inhomogen hypervaskularisierte Läsion im Lebersegment V/VI zur Darstellung. $\mathbf{b}$ In der portalvenösen Phase demarkiert sich innerhalb dieser

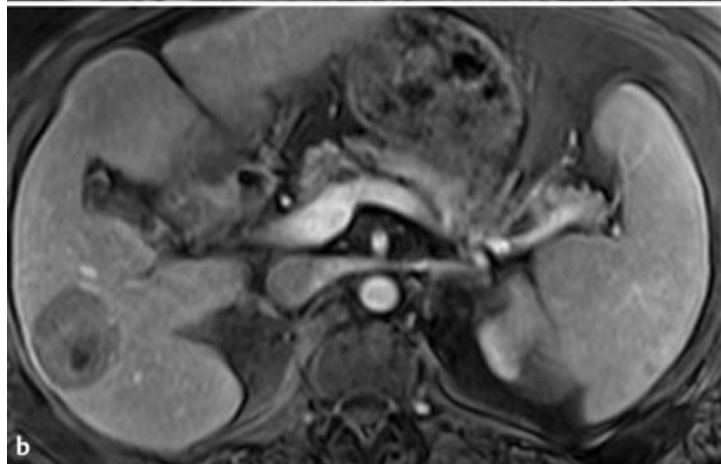
Läsion ein fettäquivalentes Areal, das einem steatotischen Areal innerhalb des HCCs entspricht.
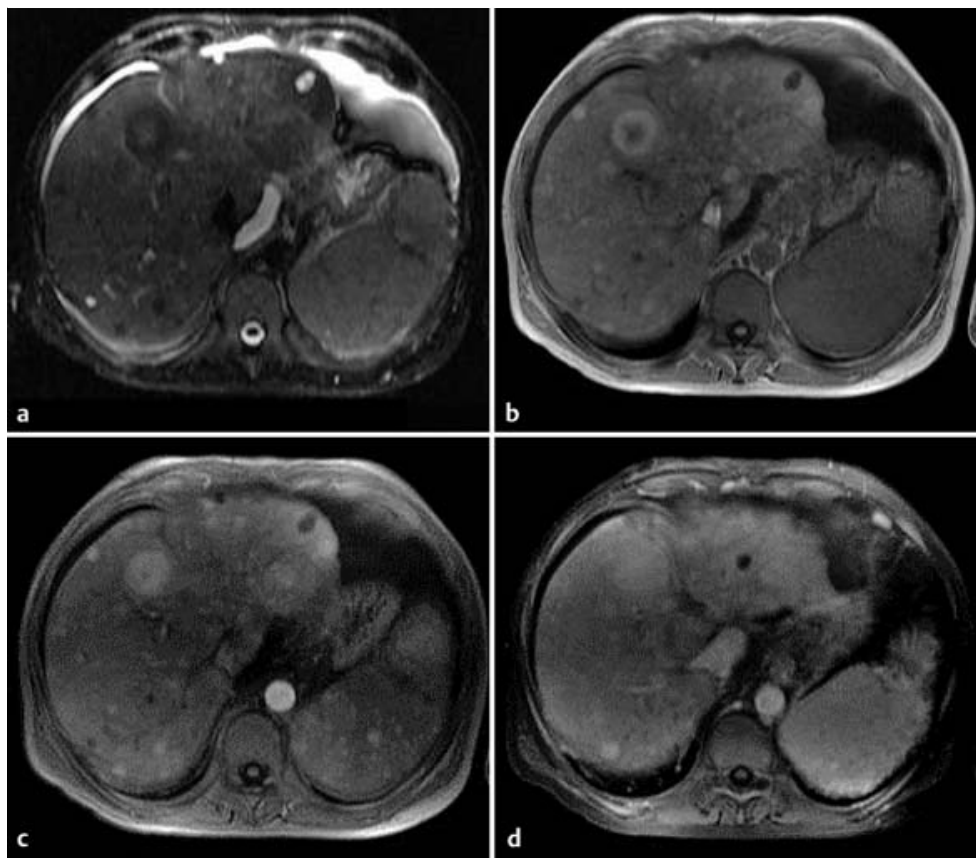

Abb. 5 Nodule in a Nodule. a, b In der nativen T2w Bildgebung zeigt sich eine hypointense Läsion mit hyperintensem Zentrum (a), die in der korrespondierenden nativen T1w Bildgebung hyperintens und mit hypointensem Zentrum zur Darstellung kommt (b). c, d Nach Kontrastmittelgabe zeigt sowohl die Peripherie als auch das Zentrum der Läsion eine Kontrastmittelaufnahme. Der Befund entspricht einem dysplastischen Knoten mit zentralem HCC, wobei das zentrale HCC noch kein typisches Auswaschen zeigt. 


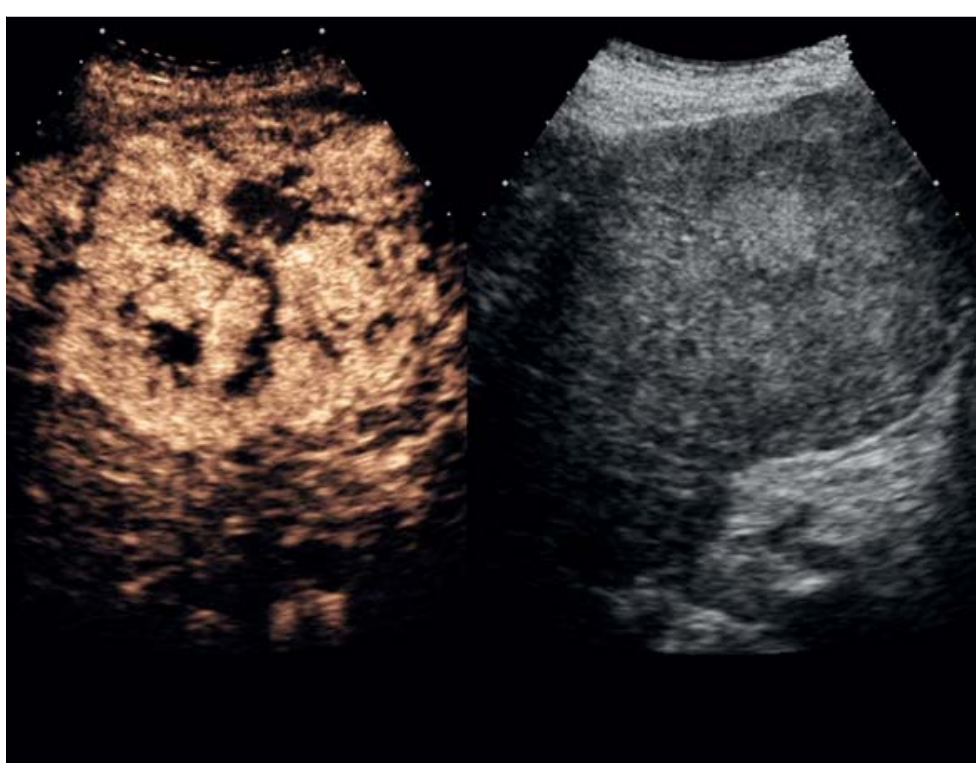

Abb. 6 Kontrastmittelverhalten des HCC im Ultraschall: Das HCC präsentiert sich im konventionellen Ultraschall zunächst als glatt berandete, rundliche Läsion mit gemischtem Echomuster (rechtes Bild). Nach Kontrastmittelgabe zeigt das HCC ein kräftiges und gegenüber dem angrenzenden Lebergewebe gesteigertes Enhancement, das die Hypervaskularisierung widerspiegelt und als Wash-in bezeichnet wird (linkes Bild).
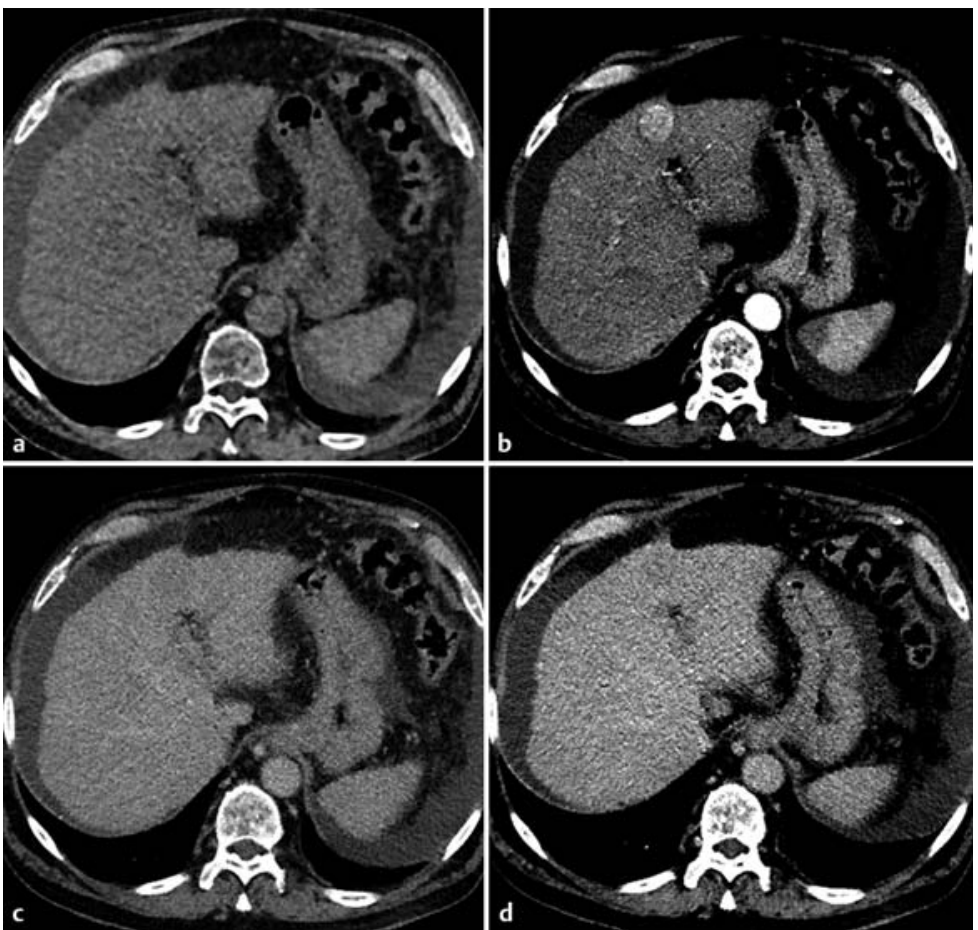

Abb. 7 Kontrastmittelverhalten des HCC in der CT. a Im Nativscan ist das HCC als isodense Raumforderung zunächst maskiert. b Nach Kontrastmittelgabe demarkiert sich das HCC in der spätarteriellen Phase als hyperdense, solitäre Läsion im Lebersegment IVa entspre-

chend einem Wash-in. c, $\mathbf{d}$ In der portalvenösen Phase und Spätphase stellt sich die Läsion hypodens und annähernd homogen entsprechend einem Wash-out dar.
- einer nativen,

- einer spätarteriellen (arteriell-dominant; ca. 15-20s nach Applikation des Kontrastmittelbolus) und

- einer portalvenösen Kontrastierungsphase unter Verwendung eines nicht ionischen Kontrastmittels hoher Jodkonzentration (370 mg Jod/ml, mindestens $80 \mathrm{ml}$ ) sowie

- ggf. der Durchführung einer zusätzlichen spätvenösen Phase (z. B. 5 min nach Kontrastmittelinjektion).

HCC-Läsionen präsentieren sich im Nativscan typischerweise hypo- bis isodens, gelegentlich auch mit Verkalkungen oder Einblutungen, in der arterielldominanten Phase hyperdens infolge der früharteriellen Hyperperfusion und in der nachfolgenden portalvenösen Phase hypodens infolge des pathologischen Wash-outs, z.T. bis hin zur Kontrastumkehr (Abb.7).

Bei einer ausgeprägten Steatose können HCCLäsionen in der nativen Bildgebung maskiert oder ggf. leicht hyperdens zur Darstellung kommen.

\section{- Magnetresonanztomografie}

Die MRT liefert durch Informationen über die Vaskularisierung und Zelldichte einer Läsion sowie durch Informationen bezüglich der Integrität der hepatozellulären Funktion und biliären Exkretion wichtige Diagnosekriterien für HCC-Läsionen [22].

Native MRT. Typische HCC-Läsionen stellen sich in der nativen Bildgebung auf einem T2w Bild hyperintens und auf einem T1w Bild hypointens dar, wobei Einblutungen, intratumorales Fett, Kupfer oder Glykogen zu einer erhöhten Signalintensität in der T1-Wichtung führen können [23] und auch gerade das frühe HCC gelegentlich isointens in der nativen T1-Wichtung zur Darstellung kommt [30].

Kontrastmittel. Wichtige zusätzliche Informationen liefern kontrastverstärkte Sequenzen unter Verwendung Gd-haltiger extrazellulärer und hepatozytenspezifischer Kontrastmittel. Analog zur CE-CT ist die HCCLäsion unter Verwendung von extrazellulärem Kontrastmittel als hypervaskularisierte Läsion in der arteriell-dominanten Phase und mit einem pathologischen Wash-out in der portalvenösen und späten Phase charakterisiert (Abb.8, Abb.9).

Von den hepatozytenspezifischen Kontrastmitteln sind derzeit nur noch die hepatobiliären Kontrastmittel mit zusätzlichen extrazellulären Eigenschaften auf dem Markt: Primovist (,gadoxetic acid“), Bayer und Multi- 
hance („gadobenate dimeglumine“, Bracco). Sie werden über aktive Transportmechanismen in funktionstüchtige Hepatozyten geschleust und renal bzw. biliär eliminiert (Multihance überwiegend renal, Primovist zu etwa gleichen Anteilen renal und biliär). Eine fehlende Kontrastmittelaufnahme in den späten Aufnahmen (ca. 20 min nach Kontrastmittelapplikation bei Primovist und $60 \mathrm{~min}$ bei Multihance) zeigt das Fehlen solcher funktionstüchtigen Hepatozyten an und gilt als Beleg für eine Entdifferenzierung des Tumors. Dass die Läsionsdetektion insbesondere für kleine HCC-Läsionen $(<2 \mathrm{~cm})$ unter Verwendung gewebespezifischer Kontrastmittel gesteigert werden kann, untermauert die Metaanalyse von Liu et al. Sie fanden, dass durch den erhöhten Tumor-Leber-Kontrast im Mittel eine Sensitivität von 0,91 (95\%-CI 0,89, 0,93) und eine Spezifität von 0,95 (95\%-CI: 0,94, 0,96) in der Detektion von HCC-Läsionen erzielt werden kann [20]. Rückschlüsse auf den Differenzierungsgrad eines HCC sind unter der Verwendung eines hepatozytenspezifischen Kontrastmittels ebenfalls möglich, da der Grad der Entdifferenzierung eines Tumors über das Ausmaß der Kontrastmittelaufnahme entscheidet [21]. Einschränkend sei erwähnt, dass eine schlechte Leberfunktion (Bilirubin $>3 \mathrm{mg} / \mathrm{dl}$ ) die Kontrastmittelaufnahme in die Hepatozyten verzögert und insgesamt vermindert, sodass bisher nicht klar ist, inwieweit der Zugewinn in der Detektionsrate auch bei diesen Patienten nachzuweisen ist.

Eine schlechte Leberfunktion (Bilirubin $>3 \mathrm{mg} / \mathrm{dl}$ ) führt zur einer geringeren und verzögerten Aufnahme leberspezifischer Kontrastmittel in die Hepatozyten.

Diffusionsbildgebung. Zusätzliche Informationen liefert die Diffusionsbildgebung (DWI, „diffusion-weighted MR imaging“): Während maligne Läsionen wie das HCC niedrige ADC-Werte aufweisen, zeichnen sich gutartige, zellarme Läsionen (z.B. Zysten, Hämangiome) durch höhere ADC-Werte im Vergleich zum normalen Lebergewebe aus [27]. Zudem war die Sensitivität der DWI in der Detektion von HCC in den letzten Jahren höher als die der konventionellen MRT [28, 29]. Dieser Zugewinn wird sicher insbesondere bei den Patienten wichtig sein, bei denen ein erhöhtes Bilirubin die Aufnahme von leberspezifischem Kontrastmittel verzögert.

\section{Diagnostische Sicherheit der Methoden}

Der Nachweis des für HCC-Läsionen charakteristischen Perfusionsverhaltens kann, wie bereits geschildert, mit CEUS, CE-CT und CE-MRT erbracht werden. Unter-
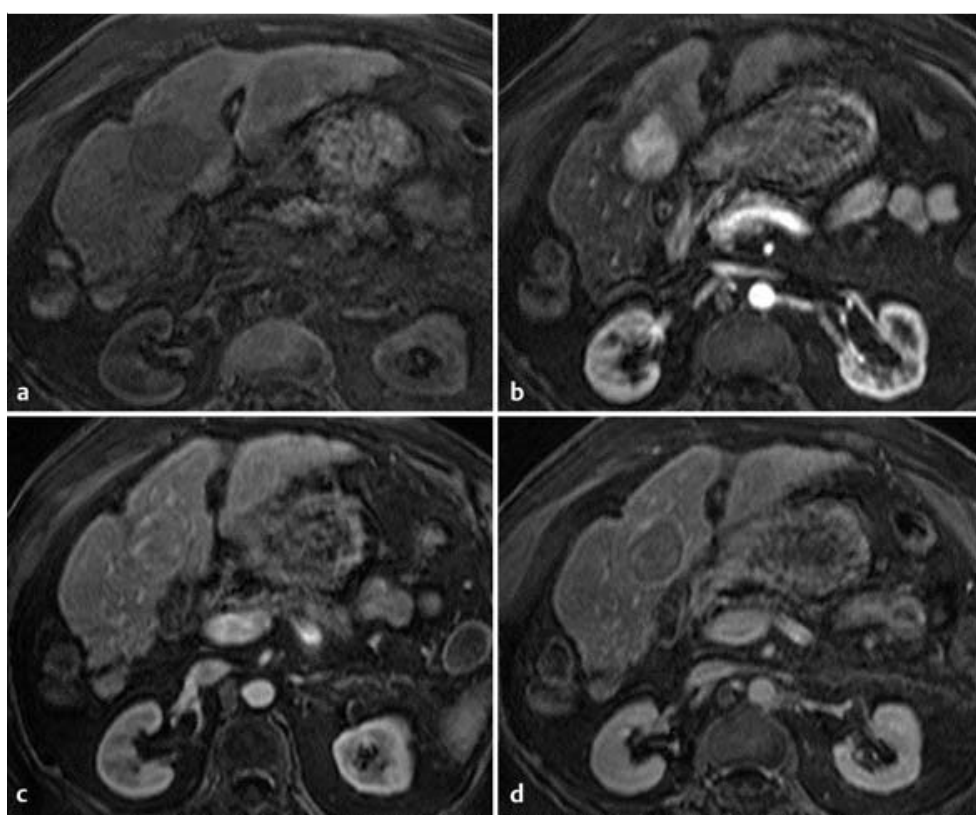

Abb. 8 Kontrastmittelverhalten des HCC in der MRT. a In der nativen T1w Sequenz ist das HCC als solitäre, glatt berandete, hypointense Raumforderung im rechten Leberlappen abgrenzbar. b Nach Kontrastmittelgabe zeigt die Läsion in der spätarteriellen Phase ein kräftiges Enhancement. c, $\mathbf{d}$ In der portalvenösen und spätvenösen Phase kommt es zum Wash-out mit Kontrastumkehr.
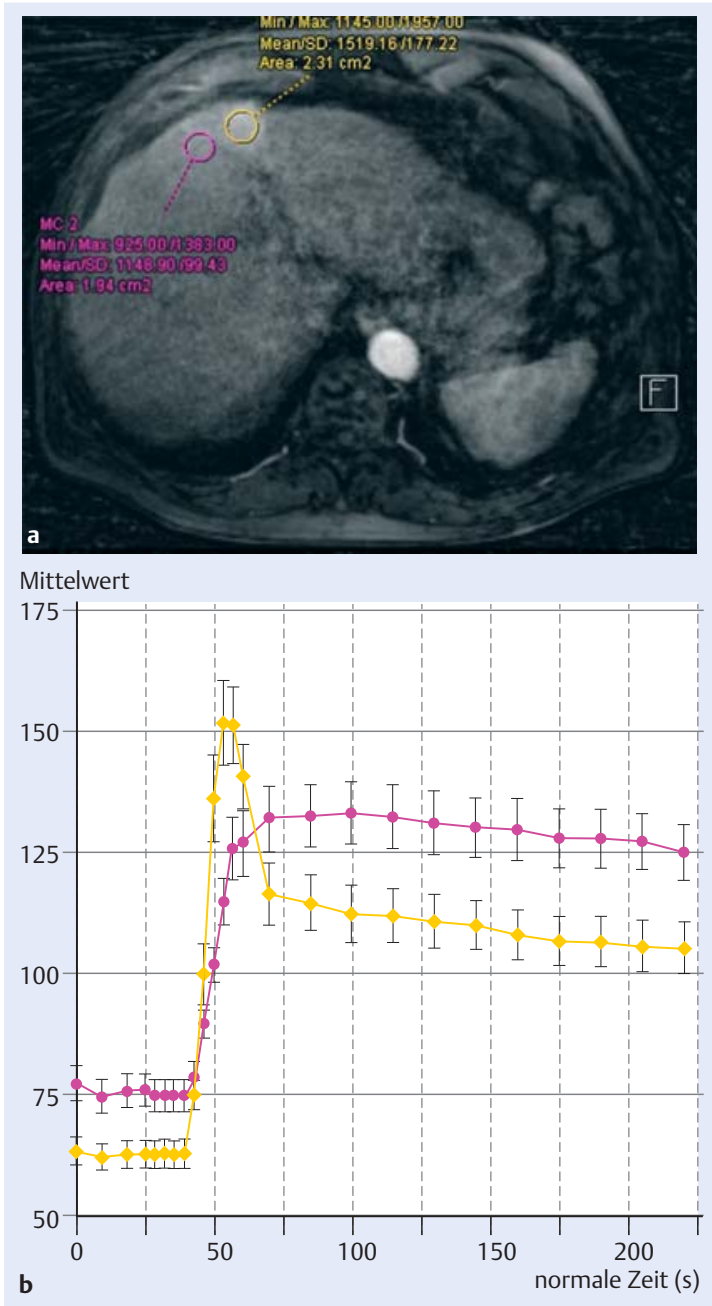

Abb. 9 Kontrastmittelverhalten eines HCC vs. Kondes angrenzenden Lebergewebes. a Bildausschnitt einer GRASP-

Sequenz (GRASP = Golden-angle Radial Sparse Parallel) mit Platzierung einer $\mathrm{ROI}$ in der HCC-

Läsion (gelb) und dem angrenzenden Lebergewebe (violett). $\mathbf{b}$ Es wird das typische Kontrastmittelverhalten eines HCC mit früherer und verstärkter Kontrastierung und schnellerem Wash-out im Vergleich zum restlichen Lebergewebe deutlich. trastmittelverhalten 
Tabelle 1

Diagnostische Sicherheit beim Nachweis von HCC.

\begin{tabular}{|c|c|c|c|}
\hline Autor, Jahr & HCC-Größe (mm) & Sensitivität (\%) & Spezifität (\%) \\
\hline \multicolumn{4}{|c|}{ Kontrastverstärkte Sonografie (nach [12-14]) } \\
\hline Leoni, 2010 & $10-30$ & 76,2 & 90,0 \\
\hline Kahalili, 2011 & $10-20$ & 53,0 & 91,0 \\
\hline Sangiovanni, 2010 & $10-20$ & 26 & 100 \\
\hline \multicolumn{4}{|c|}{ Kontrastverstärkte Computertomografie (nach [14 - 16]) } \\
\hline Baek, 2012 & $10-20$ & 80 & 97,5 \\
\hline Sangiovanni, 2010 & $10-20$ & 44 & 100 \\
\hline Seitz, 2009 & k. A. & 69,4 & 95 \\
\hline \multicolumn{4}{|c|}{ Kontrastverstärkte Magnetresonanztomografie (nach [14-16]) } \\
\hline Baek, 2012 & $10-20$ & 90 & 97 \\
\hline Sangiovanni, 2010 & $10-20$ & 44 & 100 \\
\hline Seitz, 2010 & k. A. & 82,8 & 75,5 \\
\hline
\end{tabular}

schiede bestehen jedoch hinsichtlich Sensitivität und Spezifität. Während der CEUS bei einer Sensitivität zwischen 26 und 87\% eine Spezifität zwischen 79 und $100 \%$ erreicht, liegen diese unter Verwendung der CE-CT bei $44-87 \%$ bzw. 95 - $100 \%$ und unter Verwendung der CE-MRT sogar bei $44-94 \%$ und $85-100 \%$ (Tab. 1).

Aufgrund der sowohl höheren Sensitivität als auch Spezifität der CE-CT und CE-MRT gegenüber der CEUS wird die Sonografie entsprechend der EASL-Guidelines nicht als geeignete Maßnahme gesehen, um die intrahepatische Ausbreitung zu beurteilen.

\section{Diagnostischer Algorithmus}

Durch Früherkennungsprogramme im Risikokollektiv konnte die Zahl nachgewiesener Läsionen $<2 \mathrm{~cm}$ in den letzten Jahren signifikant gesteigert werden [11]. Gerade der frühe Nachweis dieser kleinen HCC-Läsionen ermöglicht häufig ein kuratives Therapievorgehen. Dennoch sind gerade auch diese kleinen Läsionen gelegentlich ein „diagnostisches Dilemma“, weil das typische Kontrastmittelverhalten hier teilweise nicht gesehen wird. Das weitere diagnostische Vorgehen bei kleinen Läsionen ohne charakteristisches Kontrastmittelverhalten richtet sich in erster Linie nach der Tumorgröße:

- Läsionen < $1 \mathrm{~cm}$ sollten nach 3 Monaten erneut bildgebend beurteilt werden (Abb. 10).

- Bei Läsionen < $2 \mathrm{~cm}$ führt auch eine zweite, kontrastmittelgestützte Bildgebung häufig zu falsch negativen Ergebnissen, weshalb bei Läsionen dieser Größe primär eine weiterführende, bioptische Beurteilung empfohlen wird.

- Bei Läsionen $>2 \mathrm{~cm}$ ohne charakteristisches Perfusionsverhalten wird entsprechend der S3-Leitlinien primär eine zweite, kontrastmittelgestützte Bildgebung zur weiteren Beurteilung empfohlen.

\section{Wichtige Differenzialdiagnosen in der zirrhotischen Leber}

Wichtige Differenzialdiagnosen zu HCC-Läsionen in einer zirrhotischen Leber sind Regeneratknoten und dysplastische Knoten.

\section{Regeneratknoten}

Anhand von Regeneratknoten definiert man die Form der Leberzirrhose als mikronodulär ( $<3 \mathrm{~mm})$, makronodulär ( $>3 \mathrm{~mm}$ ) oder als Mischform. Regeneratknoten sind benigne Läsionen aus proliferierenden Hepatozyten mit normaler Funktion, die von fibrösen Septen umgeben sind.

MRT. Ihr Signalverhalten entspricht gewöhnlich dem des normalen Lebergewebes, weshalb sie nur schwer zu erkennen sind:

- Hämosiderinhaltige Regeneratknoten stellen sich in T1w Sequenzen hyperintens und in T2w Sequenzen hypointens dar.

- Fetthaltige Regeneratknoten zeigen sich sowohl in T1w als auch T2w Sequenzen hyperintens.

- Siderotische Knoten kommen sowohl in T1w als auch T2w Sequenzen hypointens zur Darstellung.

CT. In der nativen CT sind Regeneratknoten meist isodens, bei einer Steatosis hepatis oder im Fall eines siderotischen Knotens ggf. auch leicht hyperdens.

Sonografie. Äquivalent variiert das Erscheinungsbild der Regeneratknoten in der Sonografie (B-Bild) in Abhängigkeit vom Fettgehalt der Regenerate und vom 


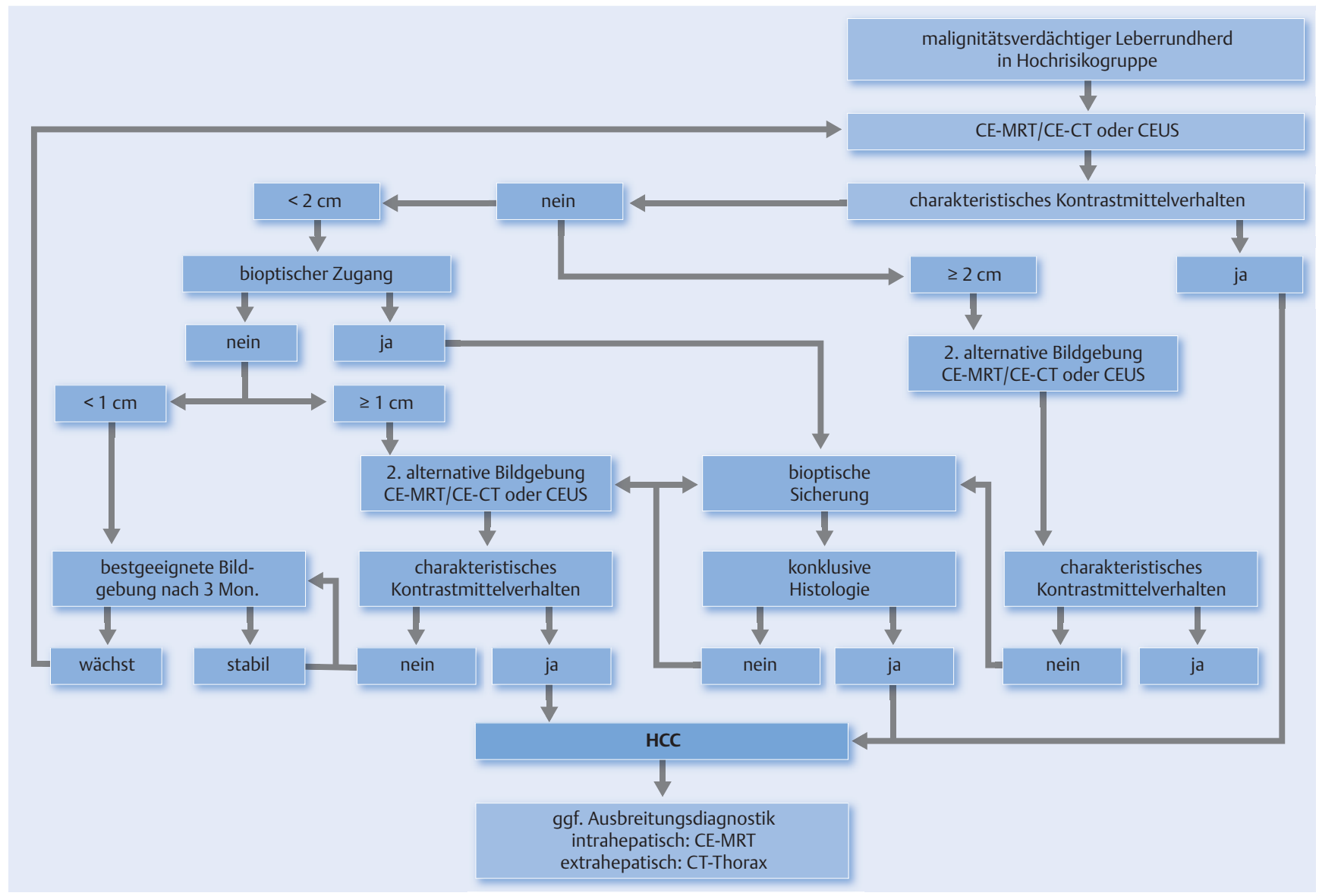

Abb. 10 Diagnostischer Algorithmus bei HCC-verdächtigen Leberläsionen gemäß aktueller S3-Leitlinie (nach [7]).

Fettgehalt sowie dem Fibrosierungsgrad des Organs zwischen echoarm und echoreich.

Da Regeneratknoten überwiegend portalvenös - und nur zu einem geringen Anteil arteriell - versorgt werden, weisen sie ein zum übrigen Leberparenchym äquivalentes Kontrastmittelverhalten in den dynamischen Sequenzen und eine homogene Kontrastierung in der hepatobiliären Phase auf.

\section{Dysplastische Knoten}

Eine Läsion wird anhand histopathologischer Kriterien als dysplastischer Knoten eingestuft. Unterschieden werden geringgradig dysplastische und hochgradig dysplastische Knoten. Auch hochgradig dysplastische Knoten zeigen noch keine eindeutigen Malignitätskriterien, man geht aber davon aus, dass viele dieser dysplastischen Knoten früher oder später in ein HCC übergehen:
- In der MRT entspricht ihr Signalverhalten in den nativen Sequenzen dem der Regeneratknoten.

- In der CT sind dysplastische Knoten schwer nachzuweisen. Sie stellen sich meist iso- bis hypodens dar.

- In der Sonografie (B-Bild) zeigt sich, äquivalent zum Regeneratknoten, eine sehr variable Echogenität (echoarm bis echoreich).

Niedriggradige dysplastische Knoten werden überwiegend portalvenös versorgt und stellen sich somit in der kontrastmittelgestützten Bildgebung isointens/isodens zum übrigen Leberparenchym dar. Hochgradig dysplastische Knoten hingegen zeichnen sich durch einen zunehmend arteriellen Zustrom mit der Folge eines arteriellen Enhancements aus. Letztlich ist aber eine sichere Unterscheidung über nicht invasive Methoden derzeit nicht möglich. 
Tabelle 2

TNM-Klassifikation des HCC (nach [17]).

\begin{tabular}{|c|c|}
\hline Einteilung & Beschreibung \\
\hline \multicolumn{2}{|l|}{ Tumor } \\
\hline TX & Primärtumor kann nicht beurteilt werden \\
\hline T0 & kein Anhalt für Primärtumor \\
\hline T1 & solitärer Tumor ohne Gefäßinvasion \\
\hline $\mathrm{T} 2$ & $\begin{array}{l}\text { solitärer Tumor mit Gefäßinvasion oder } \\
\text { multiple Tumoren, keiner mehr als } 5 \mathrm{~cm} \text { in größter Ausdehnung }\end{array}$ \\
\hline T3 & $\begin{array}{l}\text { multiple Tumoren mehr als } 5 \mathrm{~cm} \text { in größter Ausdehnung oder } \\
\text { Tumoren mit Befall eines größeren Hauptastes der V. portae oder } \\
\text { der Vv. hepaticae }\end{array}$ \\
\hline ТЗа & multiple Tumoren mehr als $5 \mathrm{~cm}$ in größter Ausdehnung \\
\hline T3b & $\begin{array}{l}\text { Tumor mit Befall eines größeren Astes der V. portae oder der } \\
\text { Vv. hepaticae }\end{array}$ \\
\hline T4 & $\begin{array}{l}\text { Tumor(en) mit direkter Invasion von Nachbarorganen, } \\
\text { ausgenommen Gallenblase oder } \\
\text { Tumor(en) mit Perforation des viszeralen Peritoneums }\end{array}$ \\
\hline \multicolumn{2}{|c|}{ Lymphknoten } \\
\hline NX & regionäre Lymphknoten können nicht beurteilt werden \\
\hline No & keine regionären Lymphknotenmetastasen \\
\hline pNO & $\begin{array}{l}\text { regionäre Lymphadenektomie und histologische Untersuchung } \\
\text { üblicherweise von } 3 \text { oder mehr LK }\end{array}$ \\
\hline N1 & regionäre Lymphknotenmetastasen \\
\hline \multicolumn{2}{|c|}{ Metastasen } \\
\hline M0 & keine Fernmetastasen \\
\hline M1 & Fernmetastasen \\
\hline
\end{tabular}

\section{Klassifikation}

Neben der Beurteilung des Tumorstadiums anhand der weltweit als Standard anerkannten TNM-Klassifikation (Tab. 2) ist das BCLC-Klassifikationssystem (BCLC= Barcelona Clinic Liver Cancer) eine gebräuchliche Form, die neben der Läsionscharakteristik auch prognostisch relevante Aspekte wie die Leberfunktion und den körperlichen Leistungszustand des Patienten berücksichtigt (Tab.3). Dieses Schema sollte deshalb als Staging in
Tabelle 3

BCLC-Klassifikation des HCC (nach [18]).

\begin{tabular}{|c|c|c|c|}
\hline BCLC & $\begin{array}{l}\text { Allgemeinzu- } \\
\text { stand Tumor }\end{array}$ & Stadium & Leberfunktion \\
\hline A 1 & ECOG 0 & $1 \mathrm{Herd}<5 \mathrm{~cm}$ & $\begin{array}{l}\text { - keine portale } \\
\text { Hypertension } \\
\text { - normales Biliru- } \\
\text { bin }\end{array}$ \\
\hline A 2 & ECOG 0 & $1 \mathrm{Herd}<5 \mathrm{~cm}$ & $\begin{array}{l}\text { - portale Hyper- } \\
\text { tension } \\
\text { - normales Biliru- } \\
\text { bin }\end{array}$ \\
\hline A 3 & ECOG 0 & $1 \mathrm{Herd}<5 \mathrm{~cm}$ & $\begin{array}{l}\text { - portale Hyper- } \\
\text { tension } \\
\text { - Bilirubin erhöht }\end{array}$ \\
\hline A 4 & ECOG 0 & $\begin{array}{l}\leq 3 \text { Herde } \\
<3 \mathrm{~cm}\end{array}$ & $\begin{array}{l}\text { Child-Pugh A } \\
\text { oder B }\end{array}$ \\
\hline B & ECOG 0 & $\begin{array}{l}\text { groß, multi- } \\
\text { lokulär }\end{array}$ & $\begin{array}{l}\text { Child-Pugh A } \\
\text { oder B }\end{array}$ \\
\hline C & ECOG $1-2$ & $\begin{array}{l}\text { Gefäßinva- } \\
\text { sion oder } \\
\text { Metastasen }\end{array}$ & $\begin{array}{l}\text { Child-Pugh A } \\
\text { oder B }\end{array}$ \\
\hline D & ECOG 3-4 & egal & Child-Pugh C \\
\hline
\end{tabular}

der Therapieeinschätzung entsprechend der aktuellen S3-Leitlinien eingesetzt werden.

\section{Das Klassifikationssystem LI-RADS (Liver Imaging} Reporting and Data System) wurde vom American College of Radiology (ACR) ins Leben gerufen. Es handelt sich dabei um ein System zur Klassifizierung nachgewiesener Leberläsionen innerhalb des Risikokollektivs. Ziel ist es, durch eine Vereinheitlichung von Terminologie und Beurteilungskriterien den Interpretationsspielraum nachgewiesener Läsionen zu verringern und somit standardisierte Informationen zu geben.

Anhand von 5 Kategorien wird die Wahrscheinlichkeit einer benignen oder malignen Läsion angegeben. Beurteilungskriterien sind neben der Vaskularisierung in der arteriellen Phase das Wachstumsverhalten, das Vorhandensein einer Kapsel bzw. Pseudokapsel sowie eines Wash-outs (Abb.11). 


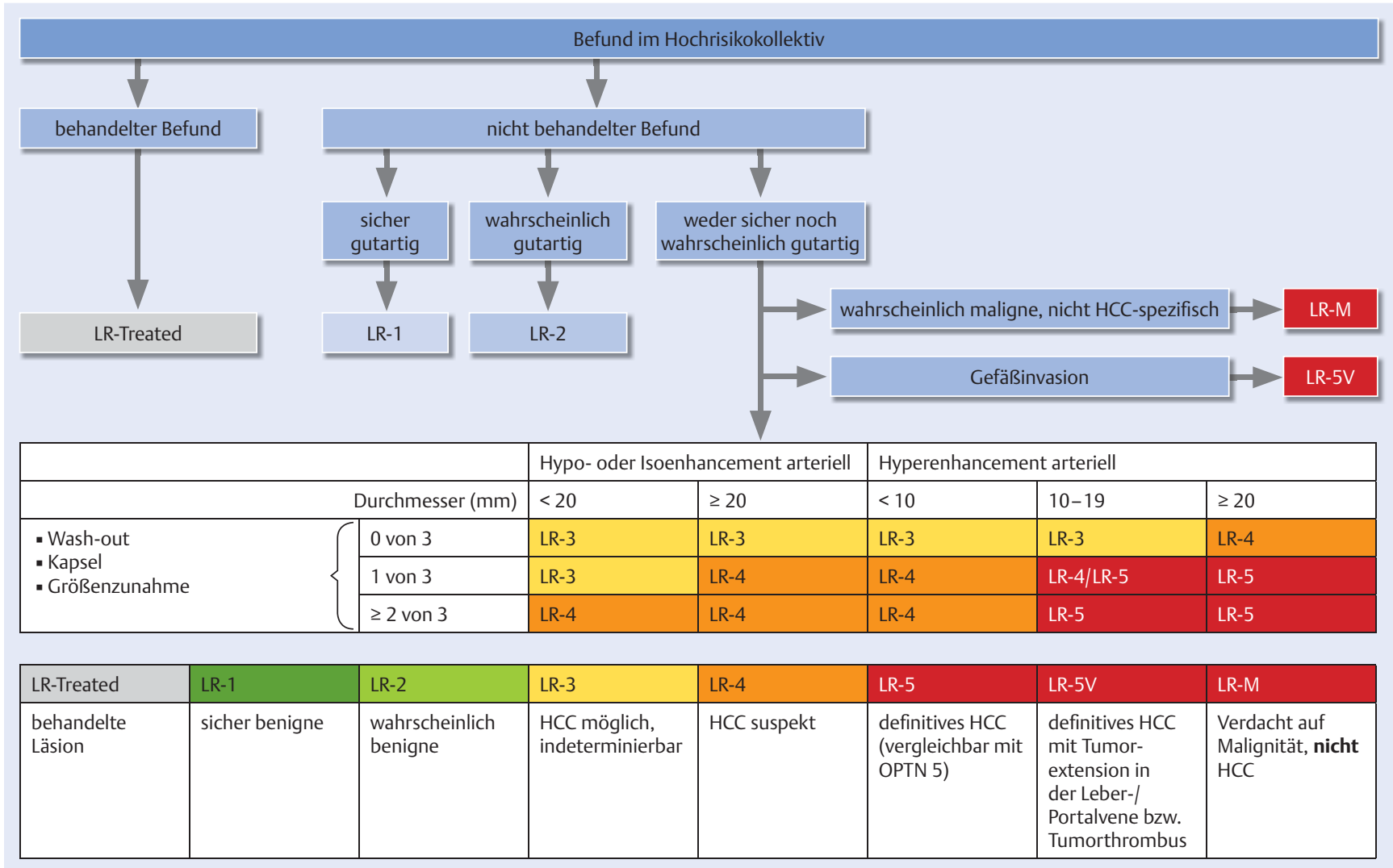

Abb.11 LI-RADS-Algorithmus zur Klassifikation nachgewiesener Leberläsionen im Risikokollektiv: Anhand der Beurteilungskriterien Wash-out, Kapsel, Größenprogredienz, Durchmesser und arterielles Hyperenhancement werden die Leberläsionen in die Gruppen LR-3 bis LR-5 bzw. LR-M und LR-5V eingeteilt, die weder sicher gutartig noch wahrscheinlich gutartig sind. Sicher gutartige und wahrscheinlich gutartige Läsionen werden den Gruppen LR-1 und LR-2 zugeordnet. „Größenprogredienz" ist definiert als Größenzunahme von $5 \mathrm{~mm}$ und mehr gegenüber der Baseline bzw. einer Größenzunahme von $>50 \%$ in $<6$ Monaten oder $>100 \%$ in > 6 Monaten (nach [19]). Die Zelle mit dem Übergang von LR-4 zu LR-5 bedeutet, dass die Befunde grundsätzlich LR-4 zugeordnet werden, es sei denn, der Durchmesser nimmt innerhalb von 6 Monaten um $\geq 50 \%$ zu (LR-5 g) oder der Befund zeigt einen Wash-out und ist als diskreter Knoten im vorausgehenden Ultraschall zu erkennen. Die untere Tabelle gibt die Definition der LI-RADS-Kategorien wieder (nach [19]).

\section{Kernaussagen}

- Das hepatozelluläre Karzinom (HCC) ist eine der häufigsten Ursachen tumorbedingter Todesfälle weltweit. Trotz der Verbesserungen in der Diagnostik und Therapie hat sich die Anzahl der Neuerkrankungen und der Todesfälle nicht verändert.

- Hauptrisikofaktoren des HCC sind Leberzirrhose (90\%), nicht alkoholische Fettleber und chronische Hepatitis-BInfektion. Weil die Diagnosestellung im Rahmen eines Früherkennungsprogramms zu deutliche höheren 5-JahreÜberlebensraten führt, werden halbjährliche Sonografien (konventioneller Ultraschall, nicht kontrastverstärkt durch entsprechend qualifiziertes Personal) beim Hochrisikokollektiv empfohlen.
- Die Diagnose eines HCC kann mit kontrastmittelunterstützten bildgebenden Verfahren wie CEUS („contrast-enhanced-ultrasound“), CE-MRI („,contrastenhanced-MRI“) und CE-CT (,contrastenhanced-CT“) gestellt werden.

- Der bildgebende Beweis eines HCC stützt sich auf ein Wash-in in der arteriell-dominanten Phase und ein Washout (teils bis hin zur Kontrastumkehr) in der folgenden portalvenösen bis parenchymatösen Phase. Diese Hauptkriterien sind jedoch nicht bei jedem HCC nachweisbar.

- Als weitere HCC-Charakteristika gelten eine Anreicherung in Tumorkapsel und Septen, ein Corona-Enhancement, eine mosaikartige Binnenstruktur, intraläsio- nales Fett und dysplastische Knoten mit fokalen Läsionen.

- Differenzialdiagnostisch kommen in einer zirrhotischen Leber Regeneratknoten und dysplastische Knoten infrage.

- Für die Klassifikation des HCC stehen die TNM-Klassifikation und die BCLC-Klassifikation (die auch prognostisch relevante Aspekte wie die Leberfunktion und den körperlichen Leistungszustand des Patienten berücksichtigt) zur Verfügung. Radiologisch kann der LI-RADSAlgorithmus zur Beurteilung von Leberläsionen eingesetzt werden (5 Kategorien mit der Angabe der Wahrscheinlichkeit einer benignen oder malignen Läsion). 


\section{Abstract}

Hepatocellular carcinoma (HCC) is one of the leading causes of cancer-related deaths worldwide. Besides the improvement in diagnostics and therapy the quantity of new cases and fatalities per year are equal. The main risk factors for HCC developing are liver cirrhosis (causing $90 \%$ of HCCs), non-alcoholic fatty liver disease and chronic hepatitis b infection. Therefore, it is recommended to perform an ultrasound screening on patients at risk every 6 month to detect HCC-lesions early. HCC can be definitely diagnosed by imaging techniques using contrast agent such as contrastenhanced-ultrasound (CEUS), contrast-enhanced-MRI (CE-MRI) and contrast-enhanced-CT (CE-CT). MRI has several advantages compared to the other modalities due to the multi-parametric approach and a higher sensitivity for tumor detection.

This article gives an overview about the typical imaging pattern of HCC in CEUS, CE-CT and CE-MRI.

\section{Keywords}

Hepatocellular carcinoma, hcc · liver cirrhosis · fatty liver disease - hepatitis b infection · contrast-enhancedultrasound · contrast-enhanced-MRI · contrast-enhanced-CT

\section{Über die Autoren}

\section{Thérèse Lincke}

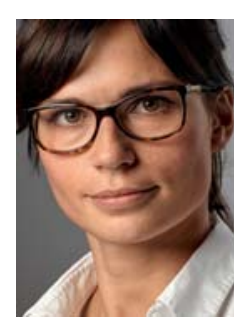

2006-2013 Studium der Medizin an der Friedrich-Schiller-Universität Jena. 2013 Promotion. 2013-2015 Assistenzärztin am Institut für Diagnostische und Interventionelle Radiologie des Kantonsspitals Aarau AG. Seit 2015 Assistenzärztin am Institut für Radiologie und Nuklearmedizin des Universitätsspitals Basel.

\section{Christoph Zech}

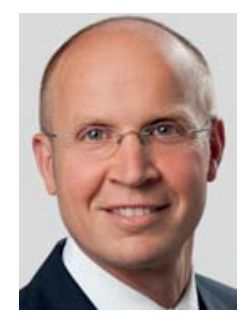

Priv.-Doz. Dr. med. Studium der Humanmedizin in Hannover, Göttingen und München. 2001 - 2007 Ausbildung zum Facharzt für Radiologie an der LMU München. 2001 Promotion. 2010 Habilitation. 2009-2012 Oberarzt am Institut für Klinische Radiologie, Klinikum der Universität München für die Bereiche MRT und Angiografie. Seit 09/2012 Leitender Arzt und Abteilungsleiter Interventionelle Radiologie an der Klinik für Radiologie und Nuklearmedizin/Universitätsspital Basel.

\section{Daniel Boll}

\section{Korrespondenzadresse}

Thérèse Lincke

Universitätsspital Basel

Klinik für Radiologie und Nuklearmedizin

Petersgraben 4

$\mathrm{CH}-4031$ Basel

Schweiz

E-Mail: therese.lincke@usb.ch

Interessenkonflikt: Christoph Zech hat von der Firma Bayer Healthcare Honorare für Vorträge erhalten. Thérèse Lincke, Daniel Boll: kein Interessenkonflikt angegeben 


\section{Literatur}

1 Estimated incidence, mortality and prevalence worldwide. WHO, International Agency on Reasearch for Cancer; 2012. Im internet: http://globocan.iarc.fr/Pages/fact_sheets_population.aspx (Stand: 2.5.2016)

2 Bruix J, Sherman M. Management of hepatocellular carcinoma: an update. Hepatology 2011; 53: 1020-1022

3 Ascha MS, Hanouneh IA, Lopez R et al. The incidence and risk factors of hepatocellular carcinoma in patients with nonalcoholic steatohepatitis. Hepatology 2010; 51: 1972 - 1978

4 Mandayam S, Jamal MM, Morgan TR. Epidemiology of alcoholic liver disease. Semin Liver Dis 2004; 24: 217 - 232

5 Lu XL et al. Risk factors for alcoholic liver disease in China. World J Gastroenterol 2004; 10: 2423-2436

6 Chang MH, Chen CJ, Lai MS. Taiwan Childhood Hepatoma Study Group. et al. Universal hepatitis B vaccination in Taiwan and the incidence of hepatocellular carcinoma in children. New Engl J Med 1997; 336: 1855 - 1859

7 Leitlinienprogramm Onkologie (Deutsche Krebsgesellschaft, Deutsche Krebshilfe, AWMF): Diagnostik und Therapie des hepatozellulären Karzinoms, Langversion 1.0. AWMF Registrierungsnummer: 032-053OL. Im Internet: http://leitlinienprogramm-onkologie.de/Leitlinien.7.0.html (Stand: 2.11.2016)

8 Llovet JM, Burroughs A, Bruix J. Hepatocellular carcinoma. Lancet 2003; 362: $1907-1917$

9 Choi JY, Lee JM, Sirlin CB. CT an MR Imaging Diagnosis and Staging of Hepatocellular Carcinoma: Part I Development, Growth, and Spread: Key Pathologic and Imaging Aspects. Radiology 2014: 272

10 Eisenhauer EA1 et al. New response evaluation criteria in solid tumours: revised RECIST guideline (version 1.1). Eur J Cancer 2009; 45: $228-247$

11 Llovet JM, Bruix J. Novel advancements in the management of hepatocellular carcinoma in 2008. J Hepatol 2008; 48: 20-37

12 Leoni $S$ et al. The impact of vascular and nonvascular findings on the noninvasive diagnosis of small hepatocellular carcinoma based on the EASL and AASLD criteria. Am J Gastroenterol 2010; 105: 599-609

13 Khalili K et al. Optimization of imaging diagnosis of $1-2 \mathrm{~cm}$ hepatocellular carcinoma: an analysis of diagnostic performance and resource utilization. J Hepatol 2011; 54: 723-728

14 Sangiovanni A et al. The diagnostic and economic impact of contrast imaging techniques in the diagnosis of small hepatocellular carcinoma in cirrhosis. Gut 2010; 59: 638-644

15 Baek CK et al. Hepatocellular carcinoma in patients with chronic liver disease: a comparison of gadoxetic acid-enhan- ced MRI and multiphasic MDCT. Clin Radiol 2012; 67: 148 1456

16 Seitz K et al. Contrast-enhanced ultrasound (CEUS) for the characterization of focal liver lesions in clinical practice (DEGUM Multicenter Trial): CEUS vs. MRI - a prospective comparison in 269 patients. Ultraschall Med 2010; 31: 492 499

17 Wittekind CH, Meyer HJ. TNM Klassifikation maligner Tumoren. 7. Aufl. Weinheim: Wiley-VCH; 2010

18 Greten TF, Manns MP. Hepatocellular carcinoma - diagnosis and treatment. Dtsch Med Wochenschr 2008; 133: 1907 1910

19 LI-RADS-algorithm. American College of Radiology; Im Internet: https://nrdr.acr.org/lirads/ (Stand: 2.11.2016)

20 Liu X, Zou L, Liu F et al. Gadoxetic Acid Disodium-Enhanced Magnetic Resonance Imaging for the Detection of Hepatocellular Carcinoma: A Meta-Analysis. PLoS ONE 2013; 8: e70896

21 Saito $\mathrm{K}$ et al. Histological grade of differentiation of hepatocellular carcinoma: comparison of the efficacy of diffusionweighted MRI with T2-weighted imaging and angiographyassisted CT. J Med Imaging Radiat Oncol 2012; 56: 261 - 269

22 Kirchner A et al. Rationale Schnittbildgebung des hepatozellulären Karzinoms. Der Radiologe 2014; 54.7: 664-672

23 Scheffel $\mathrm{H}$ et al. Praxisbuch MRT Abdomen und Becken. Heidelberg: Springer; 2012

24 Kojiro $\mathrm{M}$ et al. Pathologic diagnosis of early hepatocellular carcinoma: a report of the international consensus group for hepatocellular neoplasia. Hepatology 2009; 49.2: 658-664

25 Catalano OA et al. Differentiation of malignant thrombus from bland thrombus of the portal vein in patients with hepatocellular carcinoma: Application of diffusion-weighted MR imaging 1. Radiology 2009; 254.1: 154-162

26 Danilă M et al. Portal vein thrombosis in liver cirrhosis-the added value of contrast enhanced ultrasonography. Medical ultrasonography 2016; 18.2: 218-233

27 Kele PG, van der Jagt EJ. Diffusion weighted imaging in the liver. World J Gastroenterol 2010; 16.13: 1567 - 1576

28 Zech CJ, Reiser MF, Herrmann KA. Imaging of hepatocellular carcinoma by computed tomography and magnetic resonance imaging: state of the art. Digestive Diseases 2009; 27.2: $114-124$

29 Vandecaveye $V$ et al. Diffusion-weighted MRI provides additional value to conventional dynamic contrast-enhanced MRI for detection of hepatocellular carcinoma. Eur Radiol 2009; 19.10: $2456-2466$

30 Allkemper T. Ganzkörper-MR-Tomographie. Stuttgart: Thieme; 2006 


\section{CME-Fragen}

Die folgenden Fragen beziehen sich auf den vorangehenden Beitrag. Bitte schicken Sie uns die entsprechenden Lösungsbuchstaben. Jeweils eine Antwort ist richtig. Die Vergabe von CME-Punkten ist an die korrekte Beantwortung der Multiple-Choice-Fragen gebunden.

\section{1}

Welches ist die richtige Reihenfolg der stufenweisen Entwicklung eines progredienten HCC auf dem Boden einer zirrhotischen Leber?

\section{2}

Welches sind die 3 größten Risikofaktoren für die Entwicklung eines $\mathrm{HCC}$ ?

3

Welche der folgenden Aussagen bezüglich der Früherkennung des HCC ist richtig?
A dysplastischer Knoten, dysplastischer Knoten mit fokaler Läsion, Regeneratknoten
B dysplastischer Knoten mit fokaler Läsion, dysplastischer Knoten, Regeneratknoten
C Regeneratknoten, dysplastischer Knoten, dysplastischer Knoten mit fokaler Läsion
D dysplastischer Knoten mit fokaler Läsion, Regeneratknoten, dysplastischer Knoten
E dysplastischer Knoten, Regeneratknoten, dysplastischer Knoten mit fokaler Läsion
A Leberzirrhose, chronische Hepatitis A und chronische Hepatitis B
B chronische Hepatitis B, nicht alkoholische Fettleberhepatitis und Rauchen
C Leberzirrhose, chronische Hepatitis B und nicht alkoholische Fettleberhepatitis
D Hämochromatose, Aflatoxine und Rauchen
E Hämochromatose, Aflatoxine und Leberzirrhose

A Es gibt keine Empfehlungen bezüglich einer Früherkennung im Hochrisikokollektiv.

B Die Früherkennung des HCC erfolgt ausnahmslos mittels Sonografie.

C Die Sonografie ist zur Früherkennung von HCC-Läsionen nicht geeignet, weil die Leberzirrhose die Beurteilbarkeit der Sonografie einschränkt.

D Im Hochrisikokollektiv sollten die Patienten alle 6 Monate durch entsprechend qualifiziertes Personal sonografiert werden, um ein HCC frühzeitig nachzuweisen.

E Die Früherkennung des HCC sollte prinzipiell CT und MRT vorbehalten bleiben, weil ihre Sensitivität in der Detektion von HCC-Läsionen höher ist als die der Sonografie.
Welche der folgenden Aussagen in Bezug auf die Karzinogenese des HCC ist richtig?
A Im Zuge der Karzinogenese des HCC kommt es zu einer Zunahme der arteriellen Gefäße außerhalb der Glisson-Trias, zu einer Verringerung der Zahl der Periportalfelder und zu einer Zunahme der venösen Drainage über Lebersinusoide und Portalvenen.

B Im Zuge der Karzinogenese des HCC kommt es zu einer Zunahme der arteriellen Gefäße außerhalb der Glisson-Trias, zu einer Zunahme der Zahl der Periportalfelder und zu einer Zunahme der venöse Drainage über Lebersinusoide und Portalvenen.

C Im Zuge der Karzinogenese des HCC kommt es zu einer Zunahme der arteriellen Gefäße außerhalb der Glisson-Trias, zu einer Verringerung der Zahl der Periportalfelder und zu einer Zunahme der venöse Drainage über Lebervenen.

D Im Zuge der Karzinogenese des HCC kommt es zu einer Abnahme der arteriellen Gefäße außerhalb der Glisson-Trias, zu einer Verringerung der Zahl der Periportalfelder und zu einer Abnahme der venöse Drainage über Lebersinusoide und Portalvenen.

E Im Zuge der Karzinogenese des HCC kommt es zu einer Zunahme der arteriellen Gefäße innerhalb der Glisson-Trias, zu einer Zunahme der Zahl der Periportalfelder und zu einer Zunahme der venöse Drainage über Lebersinusoide und Portalvenen. 


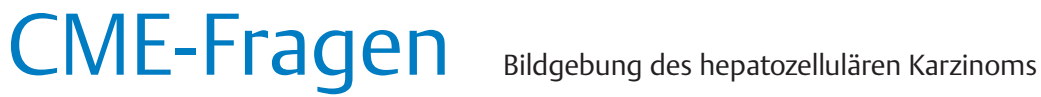

5

Welche der folgenden Aussagen ist richtig?
A Der bildgebende Nachweis des HCC ist nur mit CT und MRT möglich, weil ihre Sensitivitäten in der Detektion von HCC-Läsionen höher sind als die der Sonografie.

B Der bildgebende Nachweis des HCC ist nur mit der MRT möglich, weil ihre Sensitivität in der Detektion von HCC-Läsionen am höchsten ist.

C Der bildgebende Nachweis des HCC ist sowohl mit der kontrastverstärkten Sonografie als auch mit CT und MRT möglich.

D Der Nachweis eines HCC ist nur mit der Biopsie möglich.

E Der bildgebende Nachweis gelingt in jedem Falle mit Sonografie, CT und MRT, eine Biopsie ist nie notwendig.

\section{6}

Welches sind keine HCC-Charakteristika, die es zu überprüfen gilt?
A Corona-Enhancement
B „Nodule in a Nodule“ Appearance
C popcornartige Verkalkungen
D Tumorkapsel bzw. Pseudokapsel und intratumorale fibröse Septen
E mosaikartige Binnenstruktur

7

Welche der folgenden Aussagen bezüglich des diagnostischen Algorithmus gemäß der S3-Leitlinie von HCC-Läsionen ist richtig?
A Läsionen $>2 \mathrm{~cm}$ ohne charakteristisches Perfusionsverhalten sollten bioptisch abgeklärt werden.

B Läsionen $>2 \mathrm{~cm}$ ohne charakteristisches Perfusionsverhalten sollten nach 3 Monaten erneut bildgebend beurteilt werden.

C Läsionen $>2 \mathrm{~cm}$ ohne charakteristisches Perfusionsverhalten sollten mittels FDG-PET-CT erneut beurteilt werden.

D Läsionen $>2 \mathrm{~cm}$ ohne charakteristisches Perfusionsverhalten sollten mit einer zweiten, kontrastmittelgestützten Bildgebung weiter beurteilt werden.

E Läsionen $>2 \mathrm{~cm}$ ohne charakteristisches Perfusionsverhalten müssen nicht weiter überprüft werden.

\section{8}

Welche der folgenden Aussagen bezüglich des HCC in der MRT ist richtig?
A Die Aufnahme hepatobiliären Kontrastmittels in die Hepatozyten gilt als Beleg für die Entdifferenzierung eines Tumors.

B Eine schlechte Leberfunktion (Bilirubin $>3 \mathrm{mg} / \mathrm{dl}$ ) führt zu einer verzögerten und insgesamt verminderten Aufnahme hepatobiliären Kontrastmittels in die Hepatozyten.

C Kontrastverstärkte Sequenzen unter Verwendung von Gd-haltigen extrazellulären und hepatozytenspezifischen Kontrastmitteln liefern keine zusätzlichen Informationen in der HCCDetektion.

D Typische HCC-Läsionen stellen sich in der nativen Bildgebung auf einem T2w Bild hypointens und auf einem T1w Bild hyperintens dar.

E Einblutungen, intratumorales Fett, Kupfer oder Glykogen führen zu einer erniedrigten Signalintensität in der nativen T1w Sequenz. 


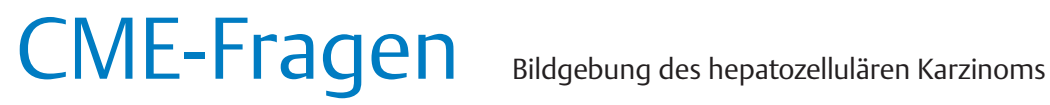

9

Welche der folgenden Aussagen bezüglich der globalen Tumorsterblichkeit ist richtig?
A Das HCC ist die zweithäufigste zum Tode führende Tumorerkrankung bei Frauen.
B Das HCC ist die zweithäufigste zum Tode führende Tumorerkrankung bei Männern.
C Das HCC ist die häufigste zum Tode führende Tumorerkrankung bei Männern.
D Das HCC ist die häufigste zum Tode führende Tumorerkrankung bei Frauen.
E Das HCC ist die häufigste zum Tode führende Tumorerkrankung bei beiden Geschlechtern.

\section{0}

Was ist die typische Reihenfolge des Perfusionsverhaltens eines HCC?
A Wash-out, Wash-in, Kontrastumkehr
B Kontrastumkehr, Wash-in, Wash-out
C Wash-out, Kontrastumkehr, Wash-in
D Kontrastumkehr, Wash-out, Wash-in
E Wash-in, Wash-out, Kontrastumkehr

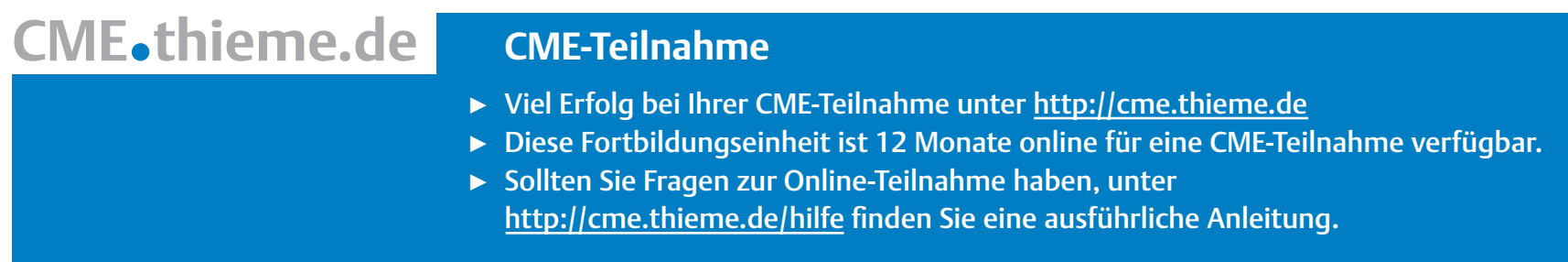

